Association for Information Systems AIS Electronic Library (AISeL)

Wirtschaftsinformatik Proceedings 2001

Wirtschaftsinformatik

September 2001

\title{
Airbus Concurrent Engineering Virtuelle Produktentwicklung am Beispiel Airbus
}

Wilfried Rieckmann

EADS Airbus GmbH, wilfried.rieckmann@airbus.dasa.de

Follow this and additional works at: http://aisel.aisnet.org/wi2001

\section{Recommended Citation}

Rieckmann, Wilfried, "Airbus Concurrent Engineering Virtuelle Produktentwicklung am Beispiel Airbus" (2001).

Wirtschaftsinformatik Proceedings 2001. 64.

http://aisel.aisnet.org/wi2001/64

This material is brought to you by the Wirtschaftsinformatik at AIS Electronic Library (AISeL). It has been accepted for inclusion in Wirtschaftsinformatik Proceedings 2001 by an authorized administrator of AIS Electronic Library (AISeL). For more information, please contact elibrary@aisnet.org. 
In: Buhl, Hans Ulrich, u.a. (Hg.) 2001. Information Age Economy; 5. Internationale Tagung Wirtschaftsinformatik 2001. Heidelberg: Physica-Verlag

ISBN: 3-7908-1427-X

(C) Physica-Verlag Heidelberg 2001 


\title{
Airbus Concurrent Engineering Virtuelle Produktentwicklung am Beispiel Airbus
}

\author{
Wilfried Rieckmann
}

EADS Airbus GmbH

\section{Entwicklung und Fertigung von Passagierflugzeugen}

Der Flugzeugbau ist gekennzeichnet durch lange und umfangreiche Entwicklungsaktivitäten. Die typische Entwicklungsdauer für ein neues Flugzeug beträgt ca. 4 Jahre. Die Fertigung der Flugzeuge erfolgt als Auftragsfertigung im Losverfahren. Durch den Boom im Flugzeugbau werden die Losgrößen allerdings ständig erhöht. Außerdem nimmt die Zahl der Flugzeuge pro Kunde ständig zu.

Der Flugzeugbau für Passagierflugzeuge ist gekennzeichnet durch ein hohes Maß an Änderungen. Die Änderungen lassen sich in 4 Kategorien einteilen:

1. Sicherheitsbedingte Änderungen

2. Fertigungsverbesserungen

3. Technische Verbesserungen

4. Kundenwünsche

Durch den hohen Änderungsumfang ist eine permanente Entwicklungstätigkeit auch an bereits in Serie gebauten Flugzeugen erforderlich. Weiterhin ist die Kontrolle des Bauzustandes der abgelieferten Flugzeuge von großer Wichtigkeit. Dies gilt umso mehr als die heutigen Flugzeuge eine Lebensdauer von ca. 40 Jahren haben. Die genannten Besonderheiten des Flugzeugbaues erfordern aus den o.g. Gründen ein effizientes Konstruktionstool sowie ein leistungsfähiges PDM-System. Weiterhin sind optimal an diese Tools angepasste Prozesse unabdingbar. Hierbei ist zu berücksichtigen, dass die Anzahl der Teile eines Flugzeuges wie die A340 ca. 2 Mio. beträgt. 


\section{Die Firma}

Airbus ist als multinationale europäische Firma der - nach Boeing - zweitgrößte Hersteller von Passagierflugzeugen $>100$ Sitzplätze. Sitz der Firma ist Toulouse/Frankreich mit Entwicklungs- und Fertigungskapazitäten in 4 europäischen Ländern (Frankreich, Deutschland, United Kingdom und Spanien).

Das Produktspektrum umfasst den Bereich:

$$
\begin{array}{ll}
\text { 100-200 Sitzplätze } & \text { A318, A319, A320, A321 } \\
\text { 200-250 Sitzplätze } & \text { mittlere Reichweite A300, A310 } \\
\text { 250-400 Sitzplätze } & \text { mittlere bis große Reichweite A330, A340 } \\
\text { 550-800 Sitzplätze } & \text { große Reichweite A380 }
\end{array}
$$

Die Endmontage der Flugzeuge erfolgt in Toulouse und Hamburg.

Die Zahl der gebauten Flugzeuge pro Jahr steigt ständig und beträgt z. Zt. ca. 300 Flugzeuge/p.a..

\section{Das Projekt ACE (Airbus Concurrent Engineering)}

Die bestehende Konkurrenz im Flugzeugbau und die Notwendigkeit Flugzeuge schneller und kostengünstiger zu entwickeln und zu fertigen, erfordert immer bessere Prozesse und Tools sowie im Falle Airbus als multinationales Unternehmen einen ständigen Zwang zu einer weiteren Harmonisierung der Arbeitsweisen und der Prozesse. Als zentraler Focalpoint zur Erarbeitung und Einführung dieser neuen Tools und Prozesse wurde das Projekt ACE geschaffen. Aufgabe des Projektes ist die Neugestaltung der Geschäftsprozesse

\section{Develop new Aircraft \\ Fulfill customer order}

Der Prozess Develop new Aircraft befasst sich mit der Neugestaltung des Flugzeugentwicklungsprozesses von der ersten Idee bis zum Erstflug des Prototypen. Der Prozess Fulfill customer order mit Entwicklung und Bau der Kundenflugzeuge. Beide Prozesse nutzen moderne 3D-CAD-Methoden und state of the art multisitefähige PDM Systeme. Das Projekt hat eine Laufzeit von 8 Jahren und beschäftigt etwa 350 Mitarbeiter.

In beiden Prozessen wird vorrangig der technische Aspekt der Prozesse betrachtet. Es werden jedoch auch alle Schnittstellen zu Beauftragungs- und Abrechnungssystemen geschaffen. Das Projekt ist airbusweit tätig und schließt alle Entwicklungsund Fertigungsstandorte der Firma Airbus mit ein.

Im Rahmen dieses Projektes werden die Entwicklungen für das neue Langstreckenflugzeug A380 getätigt, über die hier berichtet werden soll. 


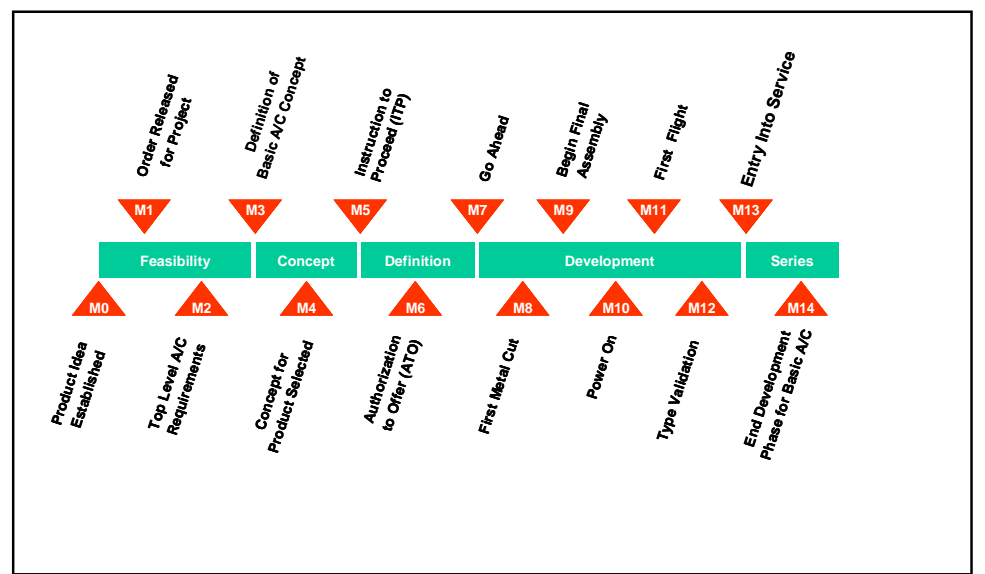

Abbildung 1: Airbus-Geschäftsprozess „Develop new Aircraft“

\subsection{Gestaltung der Prozesse}

Die Optimierung der o.g. Prozesse erfolgt nach den Prinzipien der klaren Meilensteinzuordnung und des Concurrent Engineering. Die Meilensteine des Entwicklungsprozesses werden dabei zunächst ermittelt und anschließend diesen Meilensteinen Lieferumfänge zugeordnet. Grundlage des Concurrent Engineering ist die maximal mögliche Parallelisierung der Entwicklungsarbeiten und eine mehrstufige konditionierte Freigabe der Arbeitsergebnisse.

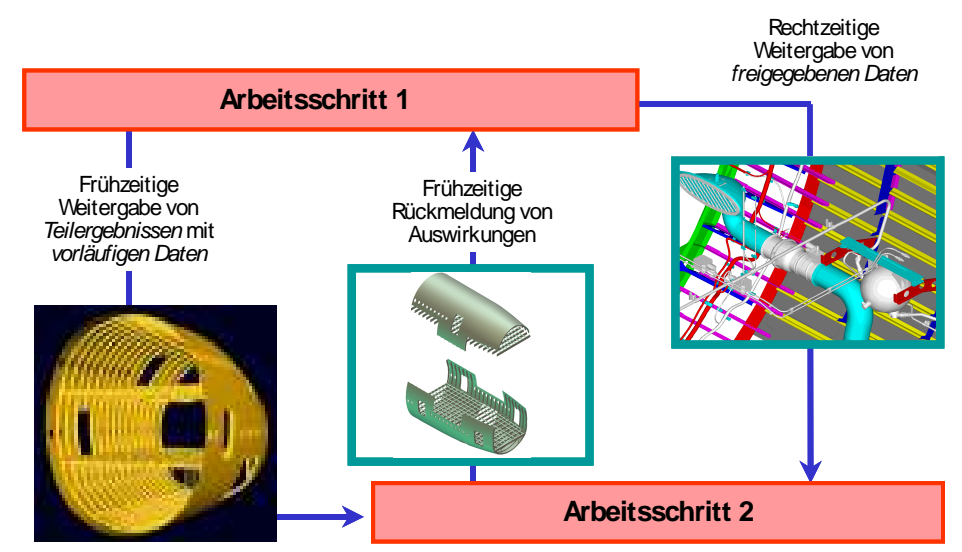

Abbildung 2: Concurrent Engineering 


\section{Virtuelle Produktentstehung}

Ein Flugzeug wie der Airbus A380 mit ca. 80m Spannweite, einem Abfluggewicht von ca. 600t und mit etwa 3Mio Einzelteilen unter Nutzung modernster Technologie, ist ohne die Verwendung moderner 3D-CAx-Tools nicht zu entwickeln. Dies schließt die Vorentwicklung, die Detailkonstruktion, die Fertigungssimulation und die eigentliche Fertigung mit ein. Bereits die ersten Entwürfe und die daraus resultierenden Windkanalmodelle werden unter Nutzung von 3D-CAx-Tools erzeugt und gefertigt. Die dabei erzeugte Präzision der Oberflächen und die verwendeten Berechnungstools ermöglichen eine bisher nicht erreichte Genauigkeit in der Vorhersage der Flugleistungen.

Für den sich anschließenden Konstruktionsprozess werden durch den Einsatz der 3D-CAx-Tools neue Arbeitsweisen möglich. Besonders zu erwähnen ist hierbei das Prinzip der Mastermodelle. Entsprechend dem Konstruktionsfortschritt unterscheidet man zwischen folgenden Mastermodellen:

\begin{tabular}{|l|l|}
\hline Mastergeometry model & Äußere Oberfläche des Flugzeuges \\
\hline Systems model & Grobe Beschreibung der Flugzeugsysteme \\
\hline Space allocation model & Beschreibung der Bauräume des Flugzeuges \\
\hline Definition model & Detaillierte Darstellung der Konstruktion \\
\hline
\end{tabular}

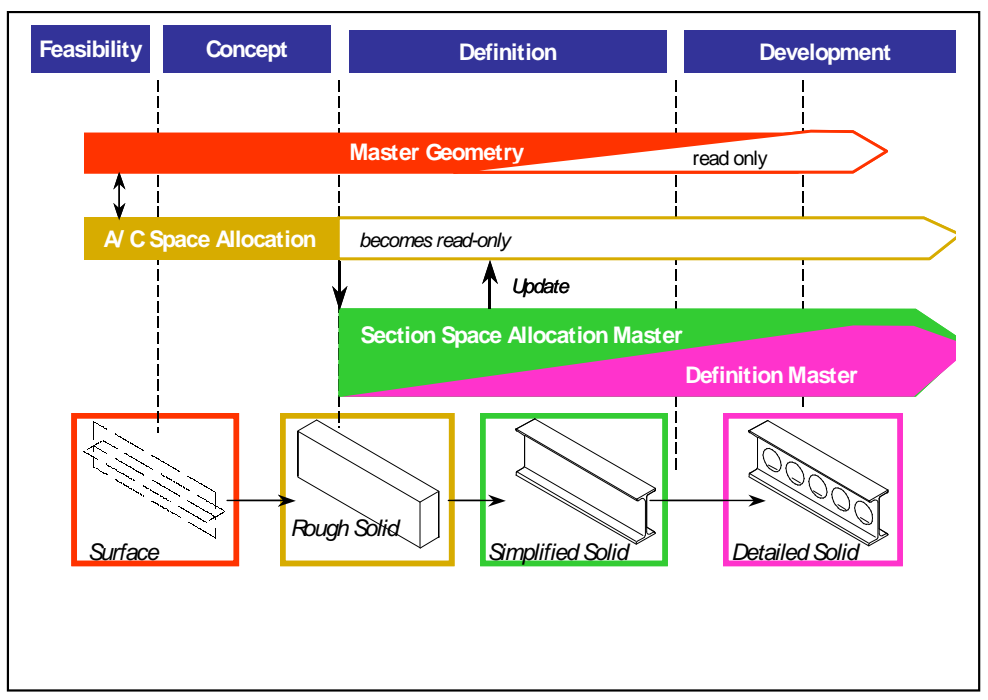

Abbildung 3: Master-Modelle 
Die virtuelle Beschreibung des Flugzeuges in dieser Form ermöglicht eine wesentlich verbesserte Kommunikation zwischen den Konstruktionbeteiligten im Hause und zu den Unterauftragnehmern. Sie ermöglicht zudem den Verzicht auf physikalische Mock-ups. Durch diese Arbeitsweise ist eine erhebliche Effizienzsteigerung in der Konstruktion möglich.

Ein weiterer Vorteil dieser Vorgehensweise ist die Informationsweitergabe an die Fertigung. Sie wird dadurch in die Lage versetzt, sehr frühzeitig mit den Informationen der Konstruktion arbeiten zu können. Durch die mehrstufige Freigabe kann zusätzlich zu einem frühen Zeitpunkt bereits mit der Definition der Vorrichtungen begonnen werden. Durch die besonderen Möglichkeiten der Visualisierung von CAD-Modellen ist darüber hinaus eine erheblich verbesserte Kommunikation zwischen Konstruktion und Fertigung gegeben, die gleichzeitig in vielfältiger weise zur besseren Erstellung von Arbeitsplänen genutzt wird. Dies gilt für alle Prozesse in der Fertigung, vorrangig aber für NC-gestützte Fertigungsabläufe.

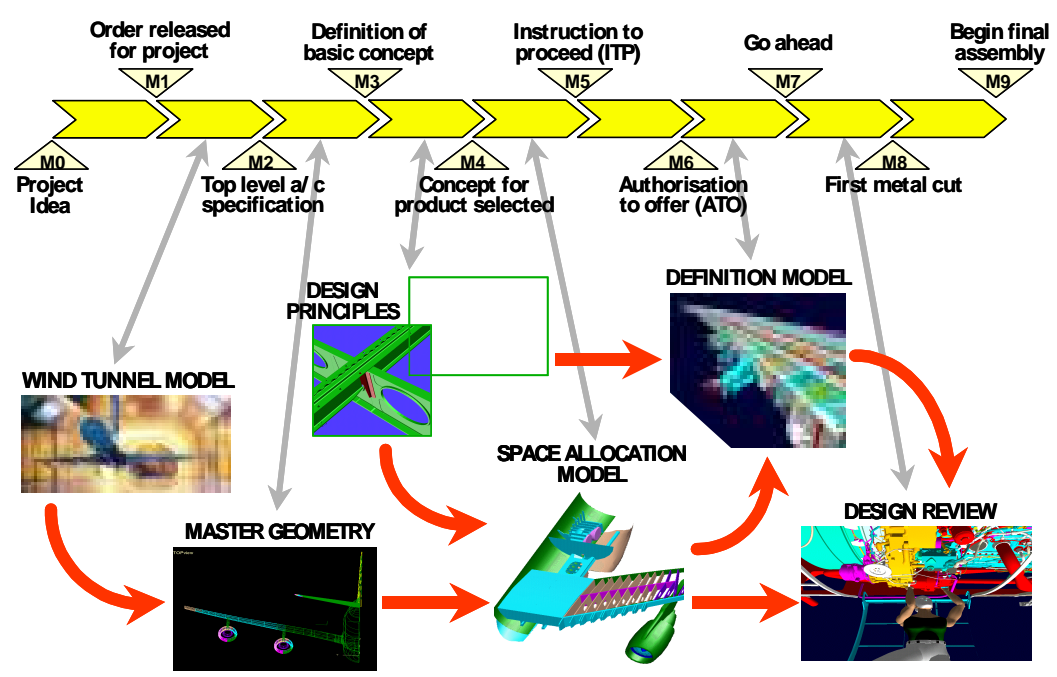

Abbildung 4: Nutzung der Master-Modelle in den Prozess-Phasen

\section{Verwendung der Produktinformationen}

Bei der Entwicklung des Flugzeuges entstehen neben den Geometrieinformationen, die direkt von der Fertigung weiterverwendet werden, vielfältige Zusatzinformationen, die die erzeugten Modelle weiter beschreiben und den Zusammenhang zwischen den Modellen wiedergeben. 
Hierbei handelt es sich um die Produktstruktur von Zusammenbauten und um nichtgeometrische Informationen zu den Bauteilen sowie um Gültigkeits- und Steuerungsinformationen zu den Bauteilen.

Die Verwaltung dieser Informationen: Geometrie, Steuerungsinformationen, Produktstrukturen, Configurationsinformationen sowie bauteilbeschreibende Informationen erfolgt in einem Product-Data-Managementsystem.

An ein PDM-System werden im Flugzeugbau erhebliche Anforderungen gestellt. Es muss in der Lage sein große Datenmengen zu verarbeiten .Für die A380 erwarten wir Datenvolumina >10 TB. Zusätzlich müssen ca. 10000 User dieses System gleichzeitig an vielen Standorten in Europa und Übersee nutzen können. Ein weiteres Kriterium muss die leichte Anpassbarkeit an die Bedürfnisse der eigenen Firma sein. Das PDM-System ist gleichzeitig die Datenbank für die Beauftragung der Fertigung, für die Erstellung der Fertigungspläne, die Abrechnungsgrundlage für die Bauteile in der Fertigung und die Bauzustandsdokumentation für Kunden und Behörden.

Für ein effizientes Arbeiten mit einem PDM-System ist die direkte Kopplung zwischen dem Geometriesystem und dem CAx-System von besonderer Bedeutung. Sie ermöglicht über die Produktstruktur des Flugzeuges die Navigation in großen und komplexen Bauräumen. Gleichzeitig können alle relevanten Geometrie- und Bauteilinformationen direkt ins PDM-System übernommen werden. Damit kann dann auch eine Bauteilfreigabe direkt aus dem Umfeld des Konstrukteurs heraus erfolgen.

Von besonderer Wichtigkeit ist dabei das Configurationsmanagement der Flugzeuge. Das CM beschreibt den Bauzustand der Flugzeuge in Bezug auf eingebaute Bauteile pro Flugzeug sowie auf eingebaute Modifikationen. Bei den Modifikationen handelt es sich um eine unter einer Nummer zusammengefassten technischen Lösung, die z.B. als Kundenwunsch in ein spezielles Kundenflugzeug eingebaut wird. Hierzu erfolgt die technische Definition in Form von CAD-Modellen und die Zusammenfassung dieser Teile im PDM-System unter einer Nummer und einer Gültigkeit des Flugzeuges. Die Erfassung der Geräte und sonstiger Zulieferteile erfolgt ebenfalls im PDM-System.

Das PDM-System stellt durch seinen umfassenden Einsatz im Flugzeugbau das Zentrale Informationssystem dar, aus dem alle produktrelevanten Informationen erzeugt werden. Es wird dabei für die Definition des Konstruktionszustands, für die Beauftragung, für die Abrechnung und für die Ermittlung des Ablieferungszustandes der Kundenflugzeuge genutzt.

Zusätzlich dient es als Medium zum Datenaustausch zwischen den vielen AirbusStandorten.

Eine wichtige Komponente des PDM-Systems in der Unterstüzung des gesamten Produktlebenszyklus im Rahmen einer system- und firmenübergreifenden Integration und Kooperation ist die Schnittstelle zum ERP-System. 
Neben den benötigten betriebswirtschaftlichen Daten sind auch die unterschiedlichen Sichten auf die Produktstruktur inklusive der anhängigen Konfigurationsdaten mit Hilfe eines unternehmensspezifischen Regelwerks auszutauschen. Sowohl das PDM- als auch das ERP-System müssen identische Punkte im Lebenszyklus des Produktes kennen, an denen mit Hilfe eines Change Control Workflows simultan die Informationen in beiden Systemen modifiziert werden.

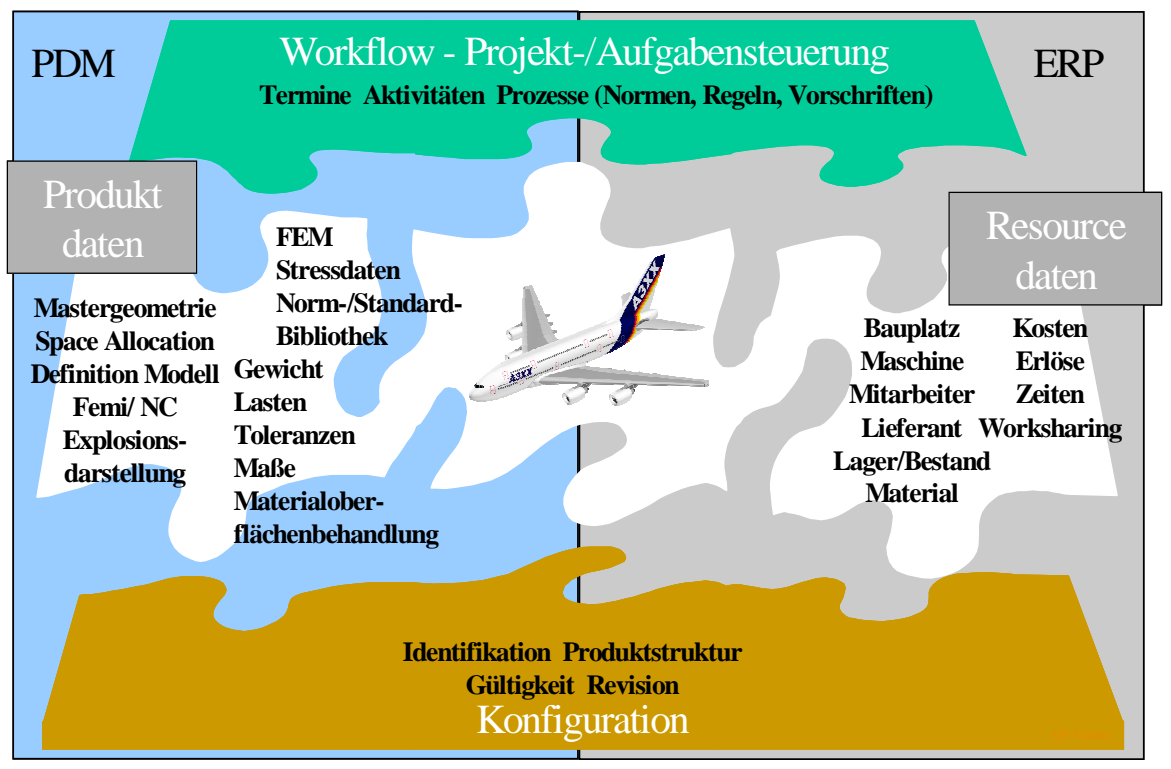

Abbildung 5: Workflow-Projekt-/Aufgabensteuerung

Eine besondere Herausforderung an den Einsatz eines neuen PDM-Systems ist die Migration von bestehenden Altsystemen in das neue PDM-System und/oder der Parallelbetrieb von Alt- und Neusystemen nebeneinander. Durch die veränderten Prozesse und den Einsatz von 3D-basierten CAx-Systemen ist eine direkte Migration oft nur schwer oder gar nicht möglich.

Deshalb ist die Einführung neuer PDM-Systeme sehr langwierig. Wir erwarten einen Zeitraum von ca. 3 Jahren bis zur vollständigen Einführung.

Im Rahmen des ACE-Projektes wird für die A380 ein sehr modernes webbasiertes PDM-System eingesetzt werden, welches zur Zeit gemeinsam mit allen Standorten entwickelt wird. 


\section{Schlussbetrachtung}

Die im Rahmen des ACE-Projektes erarbeiteten neuen Prozesse sowie die Einführung neuer 3D-CAx-Tools und eines PDM-Systems ist eine der Grundvorrausetzungen zur Entwicklung und zum Bau eines Flugzeuges von den Dimensionen einer A380.

Die Anzahl der zu konstruierenden und zu fertigenden Bauteile ist so hoch und die Verteilung der Tätigkeiten auf so viele Standorte verteilt, dass eine Steuerung des Konstruktionsprozesses ohne den Einsatz von modernen CAx- und PDM-Tools nicht möglich wäre.

Die Verwendung von Digital-Mock Up's ermöglicht eine schnelle und vollständige Weitergabe von Konstruktionsergebnissen, auf die andere Bereiche aufbauen. Weiterhin kann durch die Möglichkeiten moderner Kommunikation ein sehr schneller Informationsaustausch erreicht werden.

Das PDM-System ist dabei die zentrale Klammer für alle Informationen,die zwischen den Standorten ausgetauscht werden. Sie ermöglicht die Erkennung des $\mathrm{Zu}$ sammenhangs zwischen Bauteilen, Zusammenbauten und Flugzeugen. Sie schafft die Grundlagen für Freigaben an die Fertigungsstandorte und ermöglicht eine vereinfachte Fertigungsplanerstellung dieser Teile.

Die Nutzung dieser modernen Systeme mit all ihren Möglichkeiten ist jedoch nur dann sinnvoll und effektiv, wenn sie durch die Neugestaltung der Prozesse zielgerichtet eingesetzt werden können.

Im Zusammenspiel zwischen den Prozessgestaltern und der Einführung neuer Tools entsteht die so dringlich benötigte Kostenersparnis und Entwicklungszeitverkürzung für technische Großprojekte, wie die Entwicklung und der Bau des Passagierflugzeuges Airbus A380. 